Background (1) In 1999, the UK DOH Teenage Pregnancy Strategy Plan pledged to reduce the pregnancy rate by $50 \%$ in $<18$ yrs old by 2010 . (2) In 2009, the UK teenage pregnancy rate was 38.3 per 1,000 compared to 54.3 per 1,000 in Barking \& Dagenham (high-risk area served by the hospital)

Aim This retrospective cohort study reviewed all viable teenage pregnancies from Jan 1, 2010-Dec 31, 2010.

Method Data were obtained from the Labour Ward, Birth Notification and Operating Theatre Registries.

Results There were 257 teenagers with 260 viable babies > 28 weeks gestation. This included primigravida $(230 / 257)=89.49 \%$ and multiparous $(27 / 257)=10.51 \%$. The ages ranges from $14-19 \mathrm{yrs}$ $($ mean $=18.29 \mathrm{yrs})$. Ten $(10 / 257) 3.89 \%$ were $<16$ yrs old. There were Instrumental deliveries $(29 / 257)=11.28 \%$, Caesarean section $(36 / 257)=14.01 \%$, and Vaginal deliveries $(192 / 257)=74.71 \%$. The mean fetal birth weights were - Instrumental $3.389 \mathrm{~kg}+/-$ SD 0.468 $\mathrm{kg}$, Caesarean $3.106 \mathrm{~kg}+/-$ SD $0.752 \mathrm{~kg}$; and Vaginal Delivery 3.117 $\mathrm{kg}+/-$ SD $0.501 \mathrm{~kg}$

Maternal Morbidity Third degree tear $(\mathrm{n}=3)$, Pre-eclampsia $(\mathrm{n}=12)$ \& PPH $>1$ litre $(\mathrm{n}=4)$

Fetal Morbidity SCBU admission $(n=7)$, Stillbirth $(n=3) \&$ Shoulder dystocia $(n=2)$

Discussion During 1999-2009 the teenage pregnancy rate fell by only $13.3 \%$ in spite of the DOH Teenage Pregnancy Strategy Plan.

1. In this cohort the caesarean rate was lower $14.01 \%$ vs $24 \%$, the vaginal delivery higher $74.71 \%$ vs $65 \%$ but the instrumental was similar $11.28 \%$ vs $10 \%$ compared to the UK average (Caesarean Section Sentinel Audit).

2. There was a dedicated Teenage Pregnancy Midwifery Team providing continuity of care

3. There were $10.51 \%(27 / 257)$ multiparous teenagers thus contraceptive advice remains crucial, as UK has the highest teenage pregnancy rate in Europe

\section{PP.40 THE POTENTIAL OF DIGITAL MEDIA TO IMPROVE FETAL AND MATERNAL OUTCOMES}

doi:10.1136/archdischild-2013-303966.320

AC O'Higgins, C O'Connor, N Daly, E Kent, M Kennelly, M Turner. UCD Centre for Human Reproduction, Coombe Women and Infants University Hospital, Dublin, Ireland

Background Despite both Ireland and the United Kingdom providing free maternity care to all women, adverse fetal and maternal outcomes remain closely linked to social disadvantage and lack of support during pregnancy. A European survey found $42.4 \%$ of respondents had limited functional health literacy, closely liked to economic deprivation. Written information remains the main medium of communication for maternity services. It is likely that many of these messages are not adequately communicated to those most at risk.

Objectives This study examined the use of digital media by pregnant women to access healthcare information for pregnancy.

Methods A survey was distributed to all antenatal patients attending clinics at a large Dublin maternity hospital.

Results Of the 218 women surveyed, $81 \%$ attended public clinics and $19 \%$ attended private clinics, $60 \%$ lived in Dublin and $40 \%$ were from surrounding counties, $18 \%$ were unemployed. Overall $94 \%$ used the internet to access information about pregnancy; $100 \%$ of unemployed women use the internet to access healthcare information and $75 \%$ of women have a smartphone. Newspapers were read by only $29 \%$ of women. All women wanted some form of online/ digital support during their pregnancy, including weekly text messages about pregnancy stage-specific issues (cited by $45 \%$ ), a maternity smartphone App (44\%) and a website for feedback regarding their care (42\%).

Conclusion Digital media use is widespread across all socioeconomic groups. Healthcare communication in pregnancy should focus on digital communication channels.

\section{PP.41 MANAGEMENT OF OBESITY IN PREGNANCY IN THE WEST OF SCOTLAND}

doi:10.1136/archdischild-2013-303966.321

${ }^{1} \mathrm{P}$ Wu, ${ }^{2} \mathrm{M}$ McMillan, ${ }^{1} \mathrm{H}$ Moss, ${ }^{1} \mathrm{JL}$ Gibson. ${ }^{1}$ Southern General Hospital, Glasgow, UK; 2Princess Royal Maternity Hospital, Glasgow, UK

In 2012, a prospective 3-month audit of management of obesity in pregnancy was undertaken in Glasgow and Clyde maternity hospitals comparing practise to CMACE/RCOG guideline. ${ }^{1} 214$ women were identified out of 3,834 deliveries: $138(64 \%)$ had a booking body mass index (BMI) of 35-39 whilst 76 (36\%) had a BMI $\geq 40$. Out of total deliveries, $3.5 \%$ had a BMI of $35-39$ and $2.0 \%$ had a BMI $\geq 40$.

$43(31 \%)$ women took folic acid preconception which increased to 125 (91\%) women in first trimester. However, only 2 women took $5 \mathrm{mg}$ preconception and 7 took this during first trimester. Only 4 women had documented evidence of vitamin D supplementation. Hand-held records were available in 197 cases and 193 (98\%) women had booking BMI recorded. Anaesthetic review occurred in 68 (89\%) women with $\mathrm{BMI} \geq 40$.

Antenatal thromboprophylaxis was indicated in 43 women, but 11 women received it. Postnatally, all women with BMI $\geq 40$ should have thromboprophylaxis, however 50 (66\%) received this, out of which 14 women received appropriate dose for weight. Though only $21(10 \%)$ women had glucose tolerance test in BMI 35-39 group, this increased to $44(58 \%)$ women in BMI $\geq 40$ group.

$44(58 \%)$ women with $\mathrm{BMI} \geq 40$ had obstetric staff of specialty trainee year $\geq 6$ in attendance at delivery. There is good compliance of guideline with 195 (91\%) women having documented active management of third stage and only 1 woman induced for BMI. We conclude that some CMACE/RCOG recommendations have been implemented, though there is much scope for improvement.

\section{REFERENCE}

1. Centre for Maternal and Child Enquires/Royal College of Obstetricians and Gynaecologists Joint Guideline. Management of women with obesity in pregnancy. March 2010.

\section{PP.42 INVESTIGATION OF NEONATAL ENCEPHALOPATHY: THE LOST PLACENTAL 'BLACK BOX'}

doi:10.1136/archdischild-2013-303966.322

${ }^{3} \mathrm{D}$ Gardner, ${ }^{2} \mathrm{~A}$ Curley, ${ }^{2} \mathrm{~V}$ Venkatesh, ${ }^{3} \mathrm{MA}$ Turner, ${ }^{1} \mathrm{P}$ Clarke. 'Norfolk and Norwich University Hospitals NHS Foundation Trust, Norwich, UK; ${ }^{2}$ Cambridge University Hospitals NHS Foundation Trust, Cambridge, UK; 'iverpool Women's Hospital NHS Foundation Trust, Liverpool, UK

Background After an aeroplane crash, recovery of the 'black box' is a high priority for investigators; analysis of recorded parameters frequently identifies cause or contributing factors. The placenta likewise provides an invaluable record of the pre'crash' period in hypoxic ischaemic encephalopathy (HIE); its examination often identifies significant factors such as inflammation or vasculopathy.

Objective To determine the frequency of histopathologic placental examination and chorioamnionitis in a high-risk population of encephalopathic newborns.

Methods We studied neonates $\geq 36$ weeks' gestation admitted with HIE to three tertiary-level UK centres between 01/07/06 and $30 / 06 / 11$. We assessed if placental histopathological examination was carried out and if there was evidence of chorioamnionitis and/ or funisitis.

Results 305 infants were admitted with HIE in the 5-year study period. Placental data were unavailable for 140 outborn infants. Only 50/165 (30\%) inborn babies had placentas submitted to pathology. Histopathological examination confirmed chorioamnionitis and/or funisitis in 16/50 (32\%) cases. 